\title{
A Mesoscopic Modelling Approach for Direct Numerical Simulations of Transition to Turbulence in Hypersonic Flow with Transpiration Cooling
}

\author{
Adriano Cerminara $^{\mathrm{a}}$, Ralf Deiterding ${ }^{\mathrm{b}}$, Neil Sandham ${ }^{\mathrm{b}}$ \\ ${ }^{a}$ Faculty of Science and Engineering, Telford Innovation Campus, University of \\ Wolverhampton Telford TF2 9NT, United Kingdom \\ ${ }^{b}$ Aerodynamic and Flight mechanics Research Group, University of Southampton, \\ Southampton, Hampshire, SO17 1BJ, United Kingdom
}

\begin{abstract}
A rescaling methodology is developed for high-fidelity, cost-efficient direct numerical simulations (DNS) of flow through porous media, modelled at mesoscopic scale, in a hypersonic freestream. The simulations consider a Mach 5 hypersonic flow over a flat plate with coolant injection from a porous layer with $42 \%$ porosity. The porous layer is designed using a configuration studied in the literature, consisting of a staggered arrangement of cylinder/sphere elements. A characteristic Reynolds number $R e_{c}$ of the flow in a pore cell unit is first used to impose aerodynamic similarity between different porous layers with the same porosity, $\epsilon$, but different pore size. A relation between the pressure drop and the Reynolds number is derived to allow a controlled rescaling of the pore size from the realistic micrometre scales to higher and more affordable scales. Results of simulations carried out for higher cylinder diameters, namely $24 \mu \mathrm{m}, 48 \mu \mathrm{m}$ and $96 \mu \mathrm{m}$, demonstrate that an equivalent Darcy-Forchheimer behaviour to the reference experimental microstructure is obtained at the different pore sizes. The approach of a porous layer with staggered spheres is applied to a 3D domain case of porous injection in the Darcy limit over a flat plate, to study the transition mechanism and the associated cooling performance, in comparison with
\end{abstract}

\footnotetext{
* Corresponding author

Email address: a.cerminara@wlv.ac.uk (Adriano Cerminara)
} 
a reference case of slot injection. Results of the direct numerical simulations show that porous injection in an unstable boundary layer leads to a more rapid transition process, compared to slot injection. On the other hand, the mixing of coolant within the boundary layer is enhanced in the porous injection case, both in the immediate outer region of the porous layer and in the turbulent region. This has the beneficial effect of increasing the cooling performance by reducing the temperature near the wall, which provides a higher cooling effectiveness, compared to the slot injection case, even with an earlier transition to turbulence.

Keywords: Hypersonic flow, boundary-layer stability, wall cooling

\section{Introduction}

In hypersonic flows, where aerodynamic heating compromises the vehicle structural integrity, the film cooling technique [1, 2] can suppress the surface heat flux. In this technique, coolant is injected into the hot boundary layer 5 to form a thin film of cold fluid adjacent to the wall. We can distinguish two different injection strategies, namely effusion cooling [3, 4, in which injection occurs through localised holes, and transpiration cooling [5, 6], in which coolant transpires more uniformly through the surface of a porous coating. Due to the enhanced heat exchange occurring between the coolant and the structure through the multiple pores of micrometre dimension [6], transpiration cooling systems are potentially an efficient solution to overcome the aerodynamic heating.

In a supersonic-hypersonic flow, however, the wall cooling requirement combines with the requirement of increasing the laminar run of the boundary layer,

15 i.e. delaying transition. For this reason, injection through two-dimensional slots is in general preferred, as it reduces the 3D effects associated with hole injection [7, 8, 9. This suggests that in-depth investigation of the injection characteristics from a porous surface is needed to assess the effect of transpiration cooling systems on the transition mechanism and their suitability for the hypersonic 
flight.

Direct numerical simulations (DNS) represent a very powerful tool for capturing small-scale flow details that would be otherwise very difficult to observe and measure experimentally. On the other hand, accurate resolution of the intricate flow details in the porous region is a key requirement for a reliable assessment of transpiration cooling systems. This requires numerical simulations to be independent from simplified theoretical or empirical models that account only for a limited number of parameters and are in general constrained to specific configurations and flow conditions.

In the context of the classic theory of Darcy-Forchheimer flow through porous media, several models were developed, mostly over the first half of the twentieth century, providing a good correlation with experiments at relatively low granular Reynolds numbers (i.e. $R e_{d}$, the Reynolds number based on the particle size, lower than 150), hence in the laminar flow range. For example, among the models available in the literature describing the Darcy-Forchheimer drag imposed on the flow through a porous material, Ergun's empirical model [10, developed in 1949, is known to provide good results for certain geometrical microstructures of the porous layer, i.e. for granular media as in the case of packed beds of spheres, and dependent on the range of porosities. The coefficients defined in the model change, in general, when different geometries are considered. Another 40 factor influencing the accuracy of the model is the roughness of the solid particles, as shown by Macdonald et al. [11, in their revision of Ergun's equation. Several correlations between pressure drop and flow rate have been developed by different authors, which mostly differ in terms of the coefficients provided to weight the two contributes of the friction drag and the pressure drag, namely the Darcy and the Forchheimer drag respectively. A comprehensive list of the correlation models is provided by Erdim et al.[12, who also specify a Reynolds number range of application of Ergun's model [10. Since the late 1970's, attempts have been made to develop models suitable for turbulent flow, i.e. for high granular Reynolds numbers (e.g. $R e_{d}>300$ ), based on space and time 50 averaging of the governing equations. Some of these turbulent models and their 
following optimisations can be found in [13, 14, 15, 16, 17, 18, 19.

The difficulty in deriving a universal model of the pressure drop - flow rate correlation stands mainly in the description of the permeability $(K)$ of the interior material structure. The well-known permeability model developed in 1927 by Kozeny [20] correlates the permeability to the porosity $(\epsilon)$ and to the specific surface area per unit volume of the pores $(S)$ through a dimensionless constant $\left(c_{0}\right)$ called Kozeny constant. Even if the porosity is known for a given porous sample, an accurate estimation of the pore surface area is in general very difficult, especially for complex pore geometries. Moreover, although a value of $1 / 5$ is generally considered for the Kozeny constant, it is not universal, and will change for pores with different geometrical characteristics. A numerical study of the Kozeny constant for random packings of spheres as well as for a soil sample can be found in [21], in which it is shown that the Kozeny constant varies with the sphere radius.

The Kozeny model was then modified by Carman 22, who introduced the dependence on the average path length of the streamlines through the porous layer, i.e. the tortuosity. However, it is not simple to measure the tortuosity of a material and there is no universal law that gives the correct tortuosity given a material with certain properties. A numerical study aimed at evaluating the 70 dependence of the tortuosity from the porosity was given by Matyka et al. 23], in which the authors fitted their numerically obtained data at different porosities to four different tortuosity-porosity models provided by other authors, each one dependent on an adjustable parameter.

Hence the modelling of the pressure drop - flow rate relation through a porous structure is challenging and imposes important limitations on the applicability of studies using simplified models. On the other hand, the main challenges associated with the direct simulation approach (i.e. resolving the flow through the porous layer) can be summarised as i) the difficulty of reproducing in the computational mesh the exact internal geometry, and ii) the computational cost associated to the small micrometer scales of the interior pores, in particular in the case of direct numerical simulations. 
In the case of uniformly distributed internal porous structure, e.g. random packagings of spheres, a simplification of the computational domain is possible by identifying a main geometrical pattern which can be repeated periodically within the domain. In this fashion, different computational studies [24, 25, 26] have shown that an arrangement of staggered cylinders with constant radius in two-dimensional (2D) simulations provides in general good results in agreement with the empirical Ergun model over a wide range of porosities. In the work of Lee and Yang [25], in particular, the authors adopt a numerical model of a single pore cell in virtue of the periodicity of the flow inside a regular bank of staggered cylinders, and obtain results in a very good agreement with Ergun's model for a wide range of granular Reynolds numbers and for different porosities. However, the challenge associated with the micrometer pore dimension with regards to the computational efficiency still remains, in particular for system-level simulations aimed at representing a full-scale experimental configuration or in-flight test.

The objective of our study is to present and assess a methodology based on Lee and Yang's approach 25] which allows simulations of the flow through porous media, modelled at mesoscopic scale, in a hypersonic freestream. The aim of this mesoscopic modelling approach is to mimic the Darcy-Forchheimer behaviour of a real experimental porous material, characterised by very small pore length scales, by means of a porous layer with significantly higher pore scales that develop an equivalent Darcy-Forchheimer drag. Our approach is based on the derivation of a simple relation for the pressure drop as a function of a characteristic Reynolds number, which enables a controlled rescaling of the pore size such to obtain an equivalent Darcy-Forchheimer behaviour. Firstly, the effect of different pore sizes is assessed, in consideration of the internal Darcy-Forchheimer behaviour of the porous layer, through a comparative study between different local configurations. Then, considering flow through layers of spheres in the Darcy limit, a simulation is carried out in a large configuration, to investigate the boundary-layer transition patterns at a given blowing ratio, representative of a real experimental case. The results for the transition mechanism are then compared to a corresponding case of injection through slots, 
to evaluate the difference in the transition mechanism and associated cooling performance between slot film cooling and transpiration cooling in hypersonic flow.

\section{Numerical method}

\subsection{Governing equations}

The system of the three-dimensional dimensionless governing equations for compressible multispecies flows, written in conservation form, under the assumption of constant specific heats, is given in Cartesian coordinates as

$$
\begin{gathered}
\frac{\partial \rho}{\partial t}+\frac{\partial \rho u_{j}}{\partial x_{j}}=0, \\
\frac{\partial \rho u_{i}}{\partial t}+\frac{\partial \rho u_{i} u_{j}}{\partial x_{j}}=-\frac{\partial p}{\partial x_{i}}+\frac{1}{\operatorname{Re}} \frac{\partial \tau_{i j}}{\partial x_{j}}, \\
\frac{\partial \rho E}{\partial t}+\frac{\partial\left(\rho E+\frac{p}{\rho}\right) u_{j}}{\partial x_{j}}=\frac{1}{(\gamma-1) \operatorname{RePr} M^{2}} \frac{\partial}{\partial x_{j}}\left(\kappa \frac{\partial T}{\partial x_{j}}\right) \\
+\frac{1}{\gamma \operatorname{ReSc} M^{2}} \frac{\partial}{\partial x_{j}}\left(\rho D \sum_{k} \frac{\partial c_{k}}{\partial x_{j}} c_{p, k} T\right)+\frac{1}{\operatorname{Re}} \frac{\partial \tau_{i j} u_{i}}{\partial x_{j}} \\
\frac{\partial \rho Y_{k}}{\partial t}+\frac{\partial}{\partial x_{j}}\left(\rho Y_{k} u_{j}-\rho D \frac{\partial Y_{k}}{\partial x_{j}}\right)=0 .
\end{gathered}
$$

The terms $\rho, \rho u, \rho v, \rho w$ and $\rho E$ and $\rho Y_{k}$ are the conservative variables of the system of equations, where $\rho$ is the density, $u, v$ and $w$ are the velocity components respectively in the $x$-, $y$ - and $z$-directions, $E$ is the total energy per unit mass, $Y_{k}$ is the mass fraction of the $k$-species, and the terms $p, T, \tau_{i j}$, and $\mu$ are respectively the pressure, temperature, viscous stress tensor components and dynamic viscosity. The physical variables are normalised through their freestream reference values, except for the pressure, which is normalised with the term $\rho_{\infty}^{*} U_{\infty}^{* 2}$, and the energy, which is normalised with $U_{\infty}^{* 2}$. The superscript $\left(^{*}\right)$ is used to denote dimensional values. The characteristic length is taken as the boundary-layer displacement thickness $\left(\delta^{*}\right)$ of the similarity solution at 
the inflow. The characteristic fluid dynamic time is $\left.\delta^{*} / U_{\infty}^{*}\right)$. The terms Re, Pr, Sc, $M$, and $\gamma$ are respectively the Reynolds, Prandtl, Schmidt and Mach numbers, and the ratio of specific heats $\left(\gamma=c_{p, \infty}^{*} / c_{v, \infty}^{*}\right)$ in the freestream, i.e. the dimensionless parameters of the flow. The Reynolds number is defined as $\operatorname{Re}=\left(\rho_{\infty}^{*} U_{\infty}^{*} \delta^{*}\right) / \mu_{\infty}^{*}$; the Prandtl number is set to 0.72 , and $\gamma$ is equal to 1.4 , as the freestream gas is air. The Schmidt number is defined in terms of the mass diffusivity as $\mathrm{Sc}=\mu_{\infty}^{*} /\left(\rho_{\infty}^{*} D_{\infty}^{*}\right)$. Wilke's rule is used to express the dynamic viscosity of the mixture, as described in [27, and a power law is used to evaluate the single species viscosity. The thermal conductivity is then expressed in terms of the species viscosity, Prandtl number, specific heat and mole fraction of the $k$-species $\left(X_{k}\right)$ through the following formula,

$$
\kappa=\sum_{k} \frac{X_{k} \frac{\mu_{k} c_{p, k}}{\mathrm{Pr}_{k}}}{\sum_{l} X_{l} \phi_{k l}},
$$

with the term $\phi_{k l}$ defined as in [27. All the transport properties used in the present work are relative to a binary mixture and can be found in $[8,27,28]$.

${ }_{120}$ The system of the governing equations is closed by the relation for the total energy,

$$
E=\frac{1}{\gamma M^{2}} c_{v} T+\frac{1}{2}\left(u^{2}+v^{2}+w^{2}\right),
$$

and by the equation of state,

$$
p=\frac{1}{\gamma \mathrm{M}^{2}} \rho R T .
$$

The specific heats at constant volume and pressure, namely $c_{v}$ and $c_{p}$ respectively, as well as the gas constant $R$, represent mixture properties, which are 125 given as 8

$$
\begin{aligned}
& c_{v}=Y_{1} c_{v, 1}+Y_{2} c_{v, 2}, \\
& c_{p}=Y_{1} c_{p, 1}+Y_{2} c_{p, 2},
\end{aligned}
$$


and

$$
R=c_{p}-c_{v}
$$

It should be mentioned that the normalisation factor for the mixture gas constant and specific heats is the freestream reference value, i.e. the air gas constant, $R_{\infty}^{*}=287.058 \mathrm{~J} /(\mathrm{Kg} \mathrm{K})$. The method was validated against the numerical results of Keller et al. [8] for air-into-air injection and $\mathrm{CO}_{2}$-into-air injection over a slotted flat plate at Mach 2, obtaining a very good agreement for both cases, as reported in 37 .

\subsection{Numerical scheme}

The finite-volume method used to solve numerically the governing equations consists of a $6^{\text {th }}$-order central differencing (CD) scheme in space for both inviscid and viscous fluxes, combined with a $6^{\text {th }}$-order weighted-essentially-nonoscillatory (WENO) scheme for shock capturing, along with a $3^{r d}$-order RungeKutta method for time integration. The so-called WENO-CD scheme is provided with a switch function that turns on/off the shock-capturing scheme at discontinuities and in smooth flow regions, respectively, and has been validated over the past years for several types of compressible high speed flow configurations [29, 30, 31, 32, 33, 34.

The switch function is based on the approximate solution of the left and right states of a Riemann problem at each cell interface through Roe's averaged state, in order to locally detect the presence and orientation of shocks or steep rarefaction waves. Then, in the points where such a strong wave is found, the WENO scheme is applied, which provides numerical stability. In order to select only strong waves, while neglecting the weak ones, the method applies a threshold value to Liu's entropy condition, which is used to recognise the type of waves associated with the characteristic speeds of the left and right states [31]. In particular, the difference of the eigenvalues $u \pm a$ between the left and the right states needs to be higher than the imposed threshold value $\left(\alpha_{L i u} / a\right)$ for a wave to be considered strong enough to require the WENO method. Moreover, to increase the efficiency of the switching function, namely to minimise the use 
of the more computationally expensive WENO scheme (compared to the base central scheme), another threshold value is applied on a function based on the pressure gradient between two adjacent points, defined as

$$
\phi\left(\theta_{i}\right)=\frac{2 \theta_{i}}{\left(1+\theta_{i}\right)^{2}},
$$

with

$$
\theta_{i}=\frac{\left|p_{i+1}-p_{i}\right|}{\left|p_{i+1}+p_{i}\right|}
$$

through which the WENO method is allowed only in the high pressure gradient regions, where the function $\phi\left(\theta_{i}\right)$ is above the threshold value.

The code is also provided with a structured adaptive mesh refinement (SAMR) computational domain and the corresponding mesh are shown in figure 1, with the initial streamwise velocity field plotted in figure 1 1 . As can be seen, the porous layer is simulated with a nondimensional thickness of 1.2 and a length of 
1. It consists of equally-spaced circular elements (2D cylinders) with a radius of $r=0.078$ (corresponding to $78 \mu \mathrm{m}$ ), representing the solid particles, regularly distributed in a staggered arrangement. A $45^{\circ}$ inclination angle is formed between two adjacent staggered cylinder centres at two different $y$ locations. This configuration corresponds to a porosity of $42 \%$, which is representative of the real material porosity in the experiments of 36 .

The base grid size is $N_{x} \times N_{y}=50 \times 126$, and the domain sizes in the streamwise and vertical directions are $L_{x}=5$ and $L_{y}=10.5$, respectively, including the porous layer thickness. These grid and domain size settings provide cell sizes in the coarse (base) grid level of $\Delta x=0.1$ and $\Delta y=0.083$. The flow is initialised with the laminar boundary layer from the similarity solution. At the inflow boundary, an extrapolation boundary condition is used in the subsonic region of the boundary layer, in which the physical quantities are updated through the value of the pressure taken in the next interior cell, to account for subsonic feedback from the flow downstream. At the outlet, an outflow boundary condition (of zero-gradient) is applied, in which the quantities in the last interior cells are copied in the adjacent ghost cells. This is also used at the top boundary. A no-slip adiabatic condition is imposed at the plate wall, and a plenum condition at the bottom boundary $(y=-2)$. The plenum boundary condition consists in imposing the stagnation pressure $\left(p_{0}\right)$ and stagnation temperature $\left(T_{0}\right)$ at the bottom boundary, to simulate the presence of a underneath plenum chamber that drives vertical fluid motion in the porous layer. For the present test case, the plenum pressure and temperature were set to $p_{0}=1.5 p_{\infty}$, and $T_{0}=T_{w}$. Thus, a pressure ratio of 1.5 is imposed between the plenum chamber and the wall to generate the injection flow. 


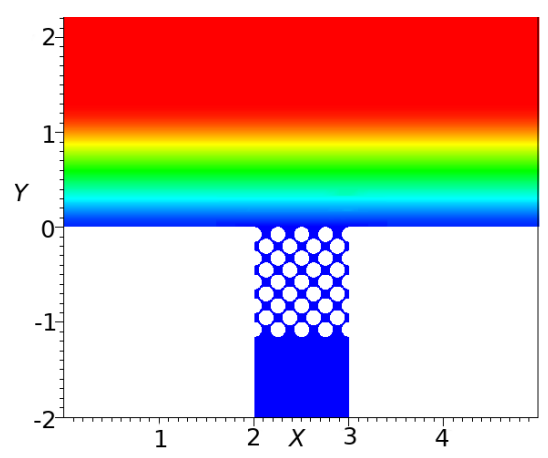

(a) $u$ field at time $t=0$

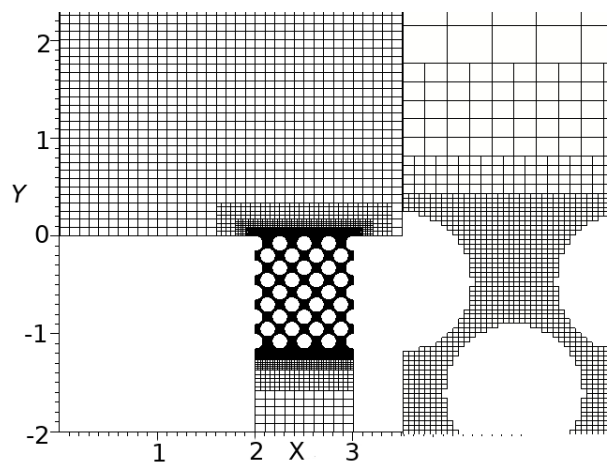

(b) mesh: domain view (left), and details around the cylinders (right)

Figure 1: Computational domain, streamwise velocity $u$ plotted (a), and corresponding mesh (b)

In figure 1 $1 \mathrm{~b}$ the different AMR levels and their localised position within the domain can be seen. Five overall levels are present, with the finer being concentrated in the region of the pores. Each finer level is embedded in the next coarser one. This arrangement of the AMR levels allow the flow features within the porous layer to be captured with optimised computational cost.

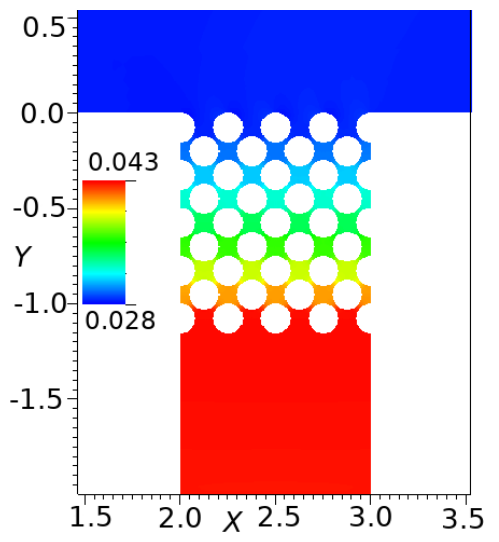

(a) pressure field

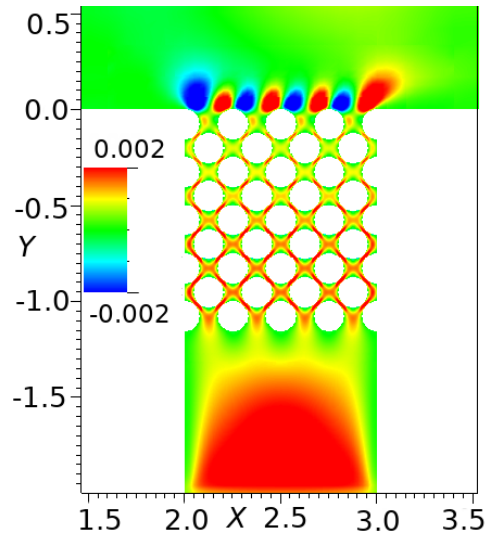

(b) vertical velocity $(v)$ field

Figure 2: Instantaneous pressure field (a), and vertical velocity field (b) 
In figure 2 qualitative instantaneous results for the pressure field and the of the vertical velocity formed just downstream of each injection jet.

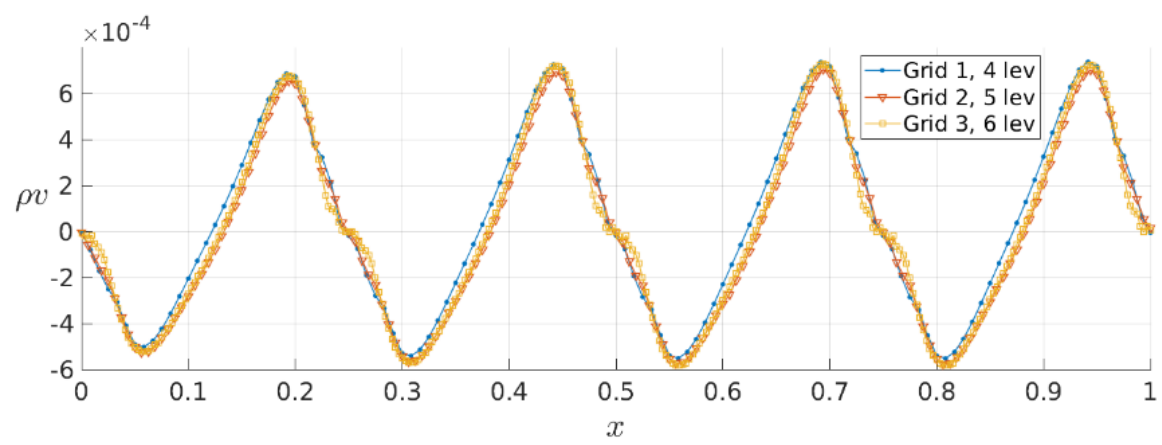

Figure 3: Grid study, on a small porous layer sample, for different AMR levels based on the blowing ratio at the surface of the porous layer $(y=0)$

\section{Mesoscopic modelling of porous material}

The aim of this section is to design a porous structure that mimics the Darcy-Forchheimer behaviour of the real material, but at considerably higher interior length scales. In order to achieve this we first follow the computational approach described by [25] for a bed of staggered cylinders, then we derive a 
relation for the pressure drop that allows rescaling of the pore length scale while maintaining the same Darcy-Forchheimer behaviour.

The relation that provides the flow rate through a porous medium given a certain pressure drop across its thickness is the so called Darcy-Forchheimer relation,

$$
\frac{\Delta p}{\delta}=\frac{\mu}{K} U+C \rho U^{2},
$$

in which $\delta$ is the thickness of the porous layer, $K$ is the material permeability, ${ }_{220} U$ is the superficial velocity averaged through the porous layer, and $C$ is a constant known as Forchheimer constant. Equation 13 can be applied over the full thickness of a porous layer, as well as on a single pore cell, provided we use the pressure drop across a pore-cell unit thickness.

Let us consider a 2D porous layer with periodic cell elements modelled as a 225 regular distribution of equal-radius cylinders in a staggered position, as the face centred structure sketched in figure 4a. As suggested by Lee and Yang 25], due to the geometrical periodicity, the dimensionless steady state solution of the flowfield between two adjacent particles along the diagonal (normalised with the pressure drop between the cylinders) in the face centred structure is the same for the other square quarters. Hence, the reference periodic fluid-dynamic domain reduces to a single quarter of the face-centred structure, as shown in figure $4 \mathrm{~b}$, which can be defined as a pore cell unit. 


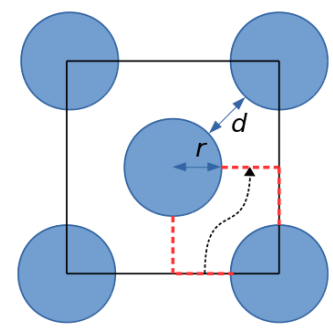

(a) face centred arrangement

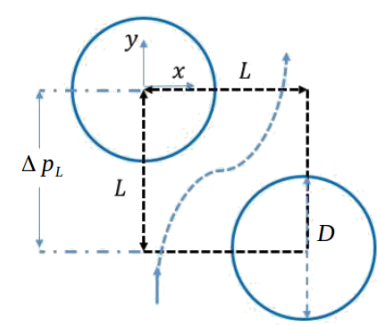

(b) periodic pore cell unit

Figure 4: Face centred cylinder arrangement (a), and pore cell unit (b)

In figure $4 \mathrm{~b}$ the reference periodic flow pattern is sketched, where $L$ is the distance between the two staggered cylinder centres, $D$ is the cylinder diameter, and $\Delta p_{L}$ is the pressure drop across the unit cell. The latter, in turn, is equal for all the unit cells, as the pressure drop across the whole thickness of the porous layer is linear. The unit-cell pressure drop $\Delta p_{L}$ can then be used as characteristic quantity to normalise the system of governing equations. Such a configuration, along with the $3 \mathrm{D}$ corresponding configuration of staggered spheres in a $3 \mathrm{D}$ domain, has been demonstrated [25, 24, 26] to provide consistent results with experiments and empirical models [10] of flow through porous samples for a wide range of Reynolds numbers.

The characteristic length in the reference system of the unit pore cell is $L$. The force per unit area that drives the flow through the pore cell is the pressure drop $\Delta p_{L}$. We can then define a characteristic fluid-dynamic velocity $U_{c}$ for this flow, which is linked to the pressure drop as

$$
U_{c}=\sqrt{\frac{\Delta p_{L}}{\rho}},
$$

where $\rho$ is the density of the flow in the pore cell. In the same way, we define the Reynolds number based on the characteristic velocity as

$$
R e_{c}=\frac{\rho U_{c} L}{\mu},
$$


where $\mu$ is the flow viscosity in the pore cell. When assigning a porosity level $(\epsilon)$, parameters of the flow are $R e_{c}$ and the ratio $D / L$ (i.e. the porosity $\epsilon$ ).

Let us consider now equation 13 , which we rewrite for a single pore cell as follows,

$$
\frac{\Delta p_{L}}{L}=\frac{\mu}{K} U+C \rho U^{2} .
$$

Note that $U$ is not equal to $U_{c}$, as $U_{c}$ represents a characteristic velocity, whereas

Reynolds number based on the cylinder diameter,

$$
R e_{d}=\frac{\rho U D}{\mu},
$$

and the Forchheimer coefficient, $F=C D$, equation 16 can be rearranged as,

$$
\frac{\Delta p_{L}}{L}=\left(\frac{D^{2}}{K}+F R e_{d}\right) \frac{\mu U}{D^{2}} .
$$

In the above equation, $D^{2} / K$ represents the Darcy drag, and $F R e_{d}$ represents the Forchheimer drag. By following Lee and Yang's [25] approach, the sum between the Darcy and the Forchheimer drag, i.e. the Darcy-Forchheimer drag $(D-F)$, can be expressed as,

$$
\left(\frac{D^{2}}{K}+F \operatorname{Re}_{d}\right)=\operatorname{Re}_{c}\left(\frac{D}{L}\right)^{2} \frac{U_{c}}{U}=\operatorname{Re}_{c}\left(\frac{D}{L}\right)^{2} \frac{1}{q},
$$

in which the term $q$ represents the average velocity along the cell outlet plane normalised with the characteristic velocity $\left(q=U / U_{c}\right)$, which can also be expressed in terms of the local velocity $v(x)$ at a generic location $x$ along the surface as,

$$
q=\frac{1}{U_{C}} \frac{1}{L} \int_{0}^{L} v d x
$$

A sketch of the velocity profile $v(x)$ on the pore surface can be seen in figure 5 


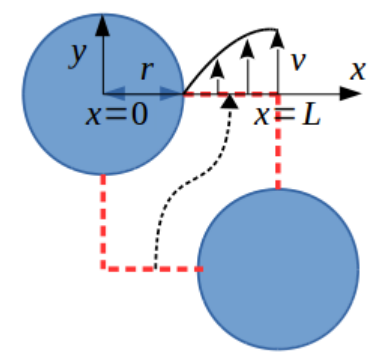

Figure 5: Sketch of the $v(x)$ velocity profile on the pore surface

The normalised velocity on the pore cell surface, $q$, represents an output from the numerical simulations, for each imposed $R e_{c}$ at a fixed porosity $\epsilon$. Then, from each computed value of $q$, the corresponding value of $R e_{d}$ can be computed through the relation

$$
R e_{d}=R e_{c}\left(\frac{D}{L}\right) q .
$$

By performing simulations in the above described dimensionless system of the pore cell unit computational domain, Lee and Yang [25] reported results in a good agreement with Ergun's [10] empirical model for the Darcy-Forchheimer drag $(D-F)$ at several values of the Reynolds number $R e_{d}$, over an extended range of porosities.

We want to compare results from our simulations with the results given in 25] at the same porosity (42\%), and at different Reynolds numbers. We consider a small periodic flat-plate domain $\left(L_{x}=4\right.$ and $\left.L_{y}=14\right)$ with a porous layer made of five rows of staggered cylinders in the absence of a hypersonic crossflow. The flow is initialised at rest in atmospheric conditions above the surface (replicating room conditions in [36], with $T_{\infty}^{*}=290 \mathrm{~K}$ ). The pressure drop imposed through the porous layer is obtained as the product between the pressure drop through a pore-cell unit $\left(\Delta p_{L}\right)$ and the overall number of cells across the thickness. The unit cell pressure drop is, in turn, obtained from the experimental value measured in [36, i.e. $\Delta p_{\exp }=\left(\Delta p^{*} / p_{\infty}^{*}\right)_{\exp }=148.5$, assuming a linear decrease of the pressure from the plenum condition $p_{0}$ to the 
surface condition $p_{\infty}$, and applying the relation

$$
\Delta p_{L}=\Delta p_{\exp } \frac{L^{*}}{\delta^{*}}
$$

In equation 22, $L^{*}=9.9 \mu \mathrm{m}$ is the pore-cell unit length and the reference length in the simulations, corresponding to a cylinder radius of $r^{*}=6 \mu \mathrm{m}$, and $\delta^{*}=5 \mathrm{~mm}$ is the thickness of the porous layer in the experiment [36]. A cell unit pressure drop of $\Delta p_{L}=0.29$ is obtained from equation 22. A ratio $D / L=1.2$ corresponds to the considered $42 \%$ porosity. The dimensional reference values for pressure and density are $\rho_{\infty}^{*}=1.225 \mathrm{~kg} / \mathrm{m}^{3}$, and $p_{\infty}^{*}=1.013 \times 10^{5} \mathrm{~Pa}$. The corresponding dimensional characteristic velocity at the outlet of the porous layer is then $U_{c}^{*}=\sqrt{\Delta p^{*} / \rho_{\infty}^{*}}=154.85 \mathrm{~m} / \mathrm{s}$. The velocity components are normalised with this value.

The Reynolds number $R e_{c}$ is varied from $R e_{c}=5$ to $R e_{c}=200$, to cover a relatively wide range of conditions. For each $R e_{c}$, the Darcy-Forchheimer drag is evaluated from the solution and applying equations 19, 20, and 21. Simulations are performed on a grid size $N_{x} \times N_{y}=210 \times 700$ with one grid level.

Figure 6 shows results for the vertical velocity field at different values of $R e_{c}$. As can be seen, at $R e_{c}=200$ an unsteady solution is obtained, as a result of the Forchheimer effect being dominant at these high values of the Reynolds number. The results of the validation study are shown in figure 7 , where the trend of the computed $D-F$ drag is plotted against different values of the Reynolds number $R e_{d}$. As can be seen, our numerical results are in a good agreement with the computational results of Lee and Yang [25] over about the whole range of Reynolds numbers. 


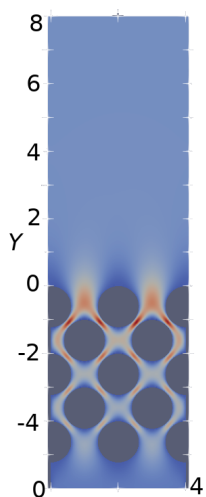

(a) $R e_{c}=25$

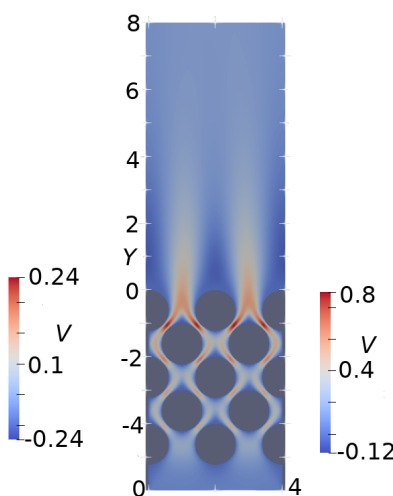

(b) $R e_{c}=100$

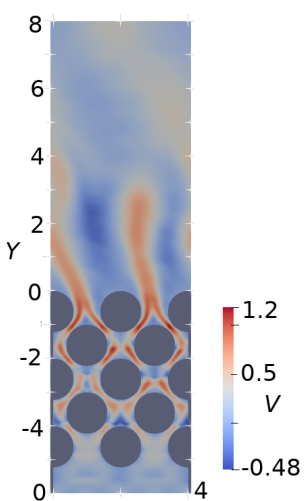

(c) $R e_{c}=200$

Figure 6: Instantaneous vertical velocity field for the case without surface crossflow, at different Reynolds numbers

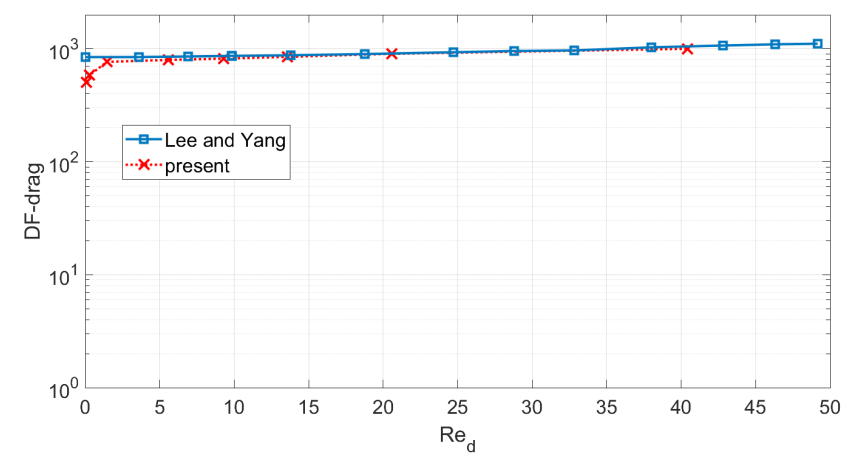

Figure 7: Result for the $D-F$ drag at different $R e_{d}$

\subsection{Equivalent D-F behaviour at different pore scales}

We carried out different simulations with different cylinder diameters, namely $D=12,24,48,96 \mu \mathrm{m}$, corresponding to increasing sizes of the pore-cell unit, with porosity fixed to $42 \%$ (i.e. $D / L=1.2$ ). We consider the flow conditions described in section 3 for a hypersonic crossflow above the porous surface. Figures 8 and 9 show computational domain and streamwise velocity field for the smallest pore-cell case and the larger cases respectively. 

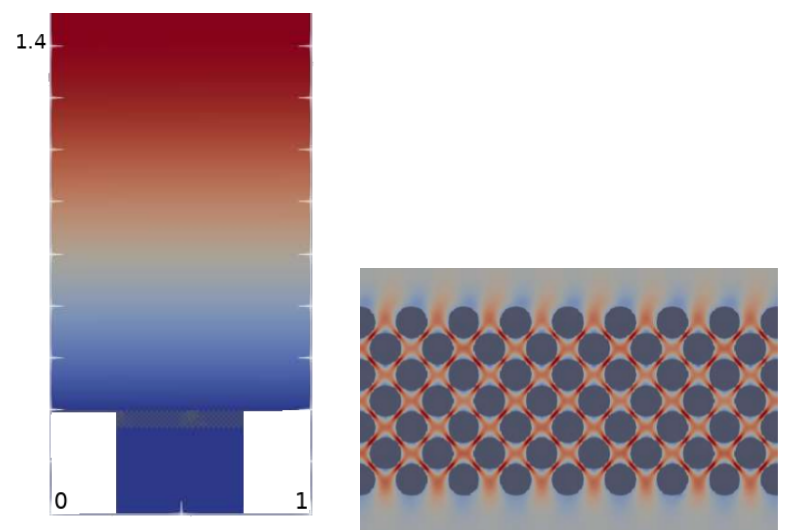

Figure 8: Streamwise velocity field (left) and close-up of $y$-momentum field in the porous layer (right), for the case $D^{*}=12 \mu \mathrm{m}$
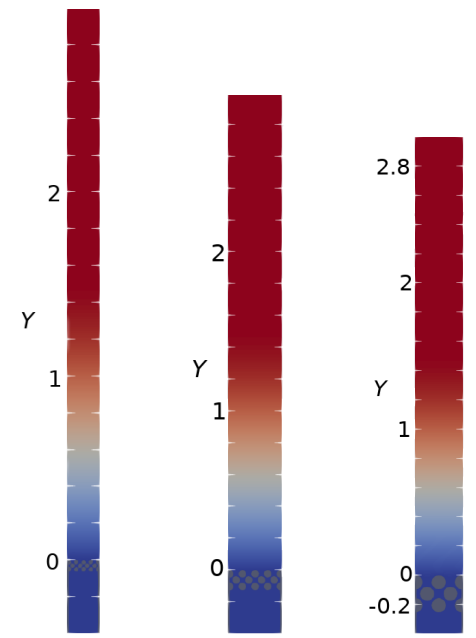
(a) $D^{*}=$
(b) $D^{*}=$
(c) $D^{*}=$
$24 \mu \mathrm{m}$
$48 \mu \mathrm{m}$
$96 \mu \mathrm{m}$

Figure 9: Periodic flat plate domains with underneath porous layer of different particle diameters. Streamwise velocity field plotted

For the smallest pore-cell case (shown in figure 8), representative of the real sample pore length scale, the pressure drop was assigned from the experimental value [36] as described in section 4.1. A blowing ratio of 0.0019 was computed at the outlet edge of the pore cells, which is very similar to the experimental 
value of 0.002 in [36], which demonstrates the validity of our numerical solution. Moreover, from the flow conditions at the wall (i.e. at the outlet of the pore cell), a characteristic velocity of $U_{c}=156.79 \mathrm{~m} / \mathrm{s}$ and a characteristic Reynolds number of $R e_{c}=1.67$ are obtained. For the larger pore-cell cases, the pressure drop was rescaled using a relation derived from equations 14 and 15 , which can be expressed as

$$
\Delta p_{L}=\rho\left(\frac{R e_{c} \mu}{\rho L}\right)^{2} .
$$

In equation 23 , the pressure drop changes with the pore-cell size $L$, while the characteristic Reynolds number is maintained constant and equal to the value obtained for the smallest pore-cell case, i.e. $R e_{c}=1.67$, thus imposing dynamic similarity between the different cases. The aim of the present simulations is to demonstrate that, by imposing dynamic similarity between cases with different pore-cell sizes through equation 23 an equivalent Darcy-Forchheimer drag can be obtained for all the cases. This, in turn, is equivalent to obtaining the same solution $q$ for all the cases, according to equation 19

From the numerical solution for each case, we compute $q$ through equation 35 20. The obtained values of $q$ are shown in table 1 for all the cases. As can be seen, a good agreement is obtained between the computed values of $q$ for the different pore-cell sizes. This is consistent with porous layers of different pore scales showing the same Darcy-Forchheimer behaviour at the considered Reynolds number, according to equation 19 , and assesses the capabilities of our mesoscopic modelling approach on the basis of equation 23 .

\begin{tabular}{|c|c|c|c|}
\hline$D^{*}=12 \mu \mathrm{m}$ & $D^{*}=24 \mu \mathrm{m}$ & $D^{*}=48 \mu \mathrm{m}$ & $D^{*}=96 \mu \mathrm{m}$ \\
$q=3.8 \times 10^{-3}$ & $q=3.7 \times 10^{-3}$ & $q=3.6 \times 10^{-3}$ & $q=3.8 \times 10^{-3}$ \\
\hline
\end{tabular}

Table 1: Obtained numerical values for the velocity $q$ 


\section{Application to a spatially-developing flow with transition}

We performed a large domain simulation representative of the UHTC experimental case of Hermann et al. 36] carried out at the lowest non-zero blowing ratio (i.e. $F=0.002$ ). The simulations are intended to replicate the flow condiflows, which were described in Section 3. The wall temperature is fixed to the room temperature, $T_{w}^{*}=290 \mathrm{~K}$. The freestream conditions are reported in table 2 .

\begin{tabular}{|c|c|c|c|c|}
\hline$M$ & $R e_{\infty, / m}^{*}(1 / \mathrm{m})$ & $T_{\infty}^{*}(\mathrm{~K})$ & $\rho_{\infty}^{*}\left(\mathrm{~kg} / \mathrm{m}^{3}\right)$ & $p_{\infty}^{*}(\mathrm{~Pa})$ \\
5 & $12.6 \times 10^{6}$ & 76.6 & 0.07979 & $1.75 \times 10^{3}$ \\
\hline
\end{tabular}

Table 2: Obtained numerical values for the velocity $q$

The computational domain (shown in figure 10) is a 3D rectangular box of dimensions $L_{x}=160, L_{y}=24, L_{z}=8$, with all the length scales normalised with the displacement thickness of the inflow similar boundary layer $\left(\delta_{0}^{*}=1\right.$ mm). A grid size of $N_{x} \times N_{y} \times N_{z}=2000 \times 300 \times 100$ is used for the coarsest level. Two overall grid levels are used, with the finest one active within the porous layer. From the simulation results, $\Delta y+=\left(\Delta y^{*} \sqrt{\tau_{w}^{*} / \rho_{w}^{*}}\right) / \nu_{w}^{*}$ is equal downstream of $x=110$. Since our focus is not resolving the fully developed turbulent field (further downstream of the domain outflow boundary), but the early nonlinear growth and transition region, these values of $\Delta y+$ suggest that the grid resolution is acceptable for our purposes. The porous layer is designed as a volume underneath the plate surface of thickness 0.5 , streamwise length of 10 and extending through the whole domain size in the spanwise direction. It is formed by three rows of spheres across the thickness with nondimensional radius $r=0.14$, in a staggered arrangement in both the $x$ and $z$ directions creating a cubic-centred structure. The entry section of the porous layer is placed at $x=55$ from the inflow. From the lower surface of the porous layer 
the computational domain extends further down up to $y=-1.28$ to simulate the presence of an underneath plenum chamber.

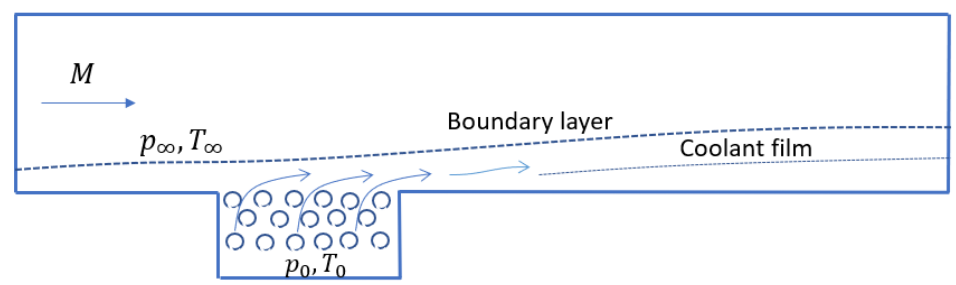

Figure 10: Sketch of computational domain

The plenum pressure imposed at the bottom boundary is $p_{0}=1.3 p_{\infty}$, which allows matching the reference experimental blowing ratio of 0.002 . It should be mentioned that this condition is no longer equivalent to matching the same characteristic Reynolds number $R e_{c}$, hence the same Darcy-Forchheimer behaviour of the porous layer in the real experimental case, which was the objective of section 4.2 This is evident by considering equation 21 and noting that when the objective is matching, for a given porosity $D / L$, the characteristic Reynolds number $R e_{c}$, hence the Darcy-Forchheimer drag by an equivalent solution $q$, as was shown in section 4.2 , the granular Reynolds number $R e_{d}$ will also be maintained constant between two configurations with different pore sizes. Hence, from the definition of granular Reynolds number (equation 17), the following relation holds between two cases with different particle diameters when matching the Darcy-Forchheimer behaviour,

$$
\left.\rho U D\right|_{\text {small }}=\left.\rho U D\right|_{\text {large }} .
$$

In other words, obtaining the same Darcy-Forchheimer behaviour between cases with different pore size and same porosity is equivalent to maintain the total mass flow discharge per pore-cell unit constant. On the other hand, from equation 24 it immediately results that the blowing ratio $\rho U$ will be different between the different pore-scale cases, i.e. mimicking the interior Darcy-Forchheimer behaviour of the porous layer does not provide the same aerodynamic effect on 
the external surface of the porous layer.

In contrast, the objective of the present section is to simulate the blowing ratio of the experimental condition, through a configuration with a larger pore

Results are also compared with a corresponding case of slot injection, which are shown in [37, in which a region of four equally-spaced injecting slots in inserted in place of the porous layer region at the same streamwise position. 
We start by observing the transition patterns in the near-wall vertical velocity field shown in figure 11 . The surface of the porous region is located between $x=55$ and $x=65$, and can be easily recognised from the higher values of the vertical velocity. A streaky structure is observed in the flowfield, which evolves rapidly as passing through the porous layer initiating the transition process. This, in turn, proceeds downstream until turbulence is reached.

The details of the porous injection are shown in the close-up in figures $12 \mathrm{a}$ and $12 \mathrm{~b}$. As can be seen, the vertical velocity field clearly shows the geometrical patterns of the porous surface in the injection region. Moreover, this figure reveals that smooth, gradually growing streaks are present upstream of the porous injection region, whereas just downstream of the porous region the streaks appear distorted and with higher amplitude, with evidence of formation of secondary structures inducing the breakdown process. This is more evident in figures $12 \mathrm{~b}$ and $12 \mathrm{c}$, which show vertical velocity within a smaller scale range and the streamwise velocity, both in grey-scale in order to highlight details of the smaller wavelength structures. In particular, figure $12 \mathrm{~b}$ shows the presence of small-wavelength oscillations in the transversal direction of the side streaks, in the region $x=65-70$, thus suggesting generation of secondary instabilities, as well as formation of a large structure at the midspan, whereas figure $12 \mathrm{c}$ shows evidence of streak bifurcation downstream of $x=70$ with formation of secondary streaks.

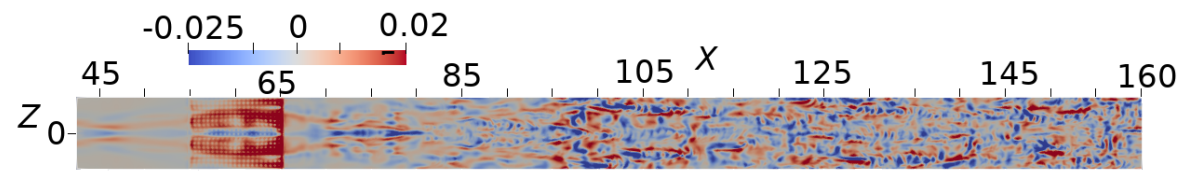

Figure 11: Near-wall vertical velocity. Top view 


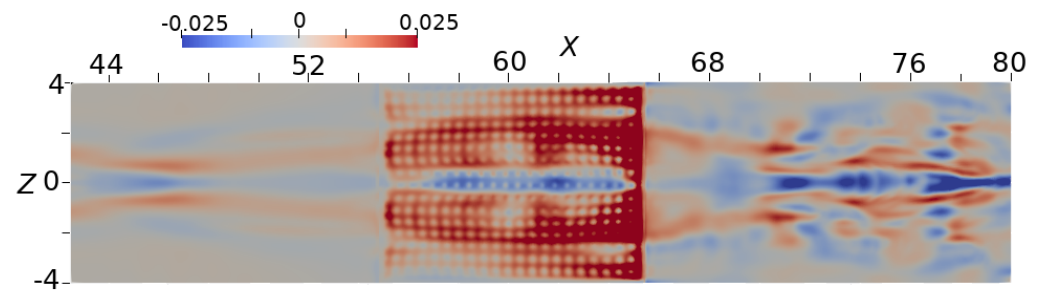

(a) v - porous injection pattern details

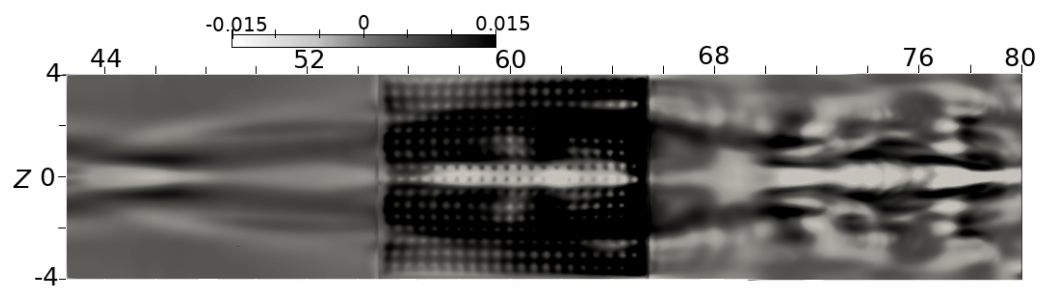

(b) v - details of downstream secondary structures

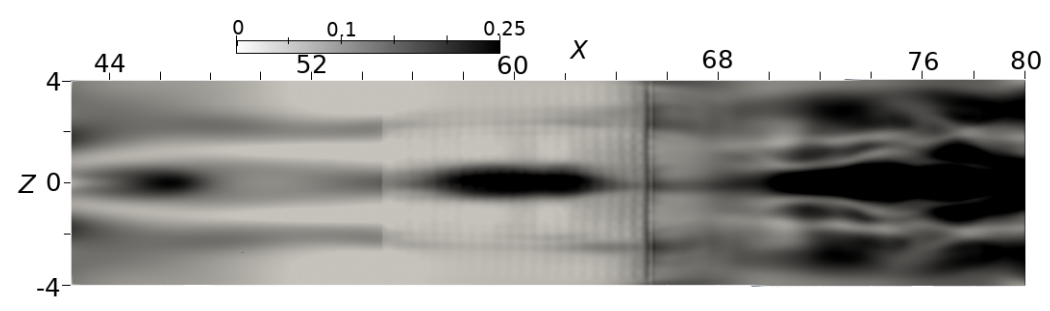

(c) $\mathrm{u}$ - details of downstream streak bifurcation

Figure 12: Close-up of near-wall vertical (a) and streamwise velocity (b) around the porous region. Top view. Grey-scale contours are used to highlight small-scale patterns

Thus, results show that a rapid nonlinear breakdown process is initiated from the porous injection region, whose source can be found by observing the associated features of the pressure field within the porous region, as shown in figure 13 First, the start of the injection region corresponds to a pronounced increase of pressure, as a result of the viscous interaction effect associated with the thickening of the boundary layer due to injection. Furthermore, the streaks in the porous region appear to be affected by narrow inclined disturbances. These disturbances appear to be associated to a system of small-wavelength pressure waves (as evident from the close-up in figure 13b originating along 
the midspan axis, which travel both towards the positive and the negative $z$ coordinates at the local Mach angle.

The system of pressure waves, which appear to be responsible for the rapid nonlinear growth of the streaks, may have different causes. One possibility may be represented by an early excitation of an unstable mode along the porous injection surface. However, the potential effect associated with the solid spheres of the porous layer should be taken into consideration. In particular, the size of the spheres can have an important influence on the local flow features above the porous surface, and, in the present case, might have played a role in the nonlinear growth of the unstable disturbances. If this is the case, it would represent an important limit on the multi-scale approach, however we note that the wavelength of the pressure waves is approximately $1 \mathrm{~mm}$ (for example, it is $0.954 \mathrm{~mm}$ if we consider the first two wave fronts from the left in figure 13b), whereas the pore spacing/wavelength is $0.48 \mathrm{~mm}$ (taken as the distance between two adjacent sphere centres), which is half the wavelength of the pressure waves. This suggests that the pressure waves are an excited feature associated with a subharmonic of the pore spacing, rather than a direct consequence of the individual pores. These waves do not appear to be local first or second unstable mode waves. The streamwise wavelength of the most unstable mode at this 465 location (imposed as forcing within the boundary layer), which was found to be a 3D first mode, is about $31 \mathrm{~mm}$, whereas the wavelength of the least stable local 2D second mode was found approximately equal to $6 \mathrm{~mm}$, hence still much higher than the wavelength of the observed pressure waves. 


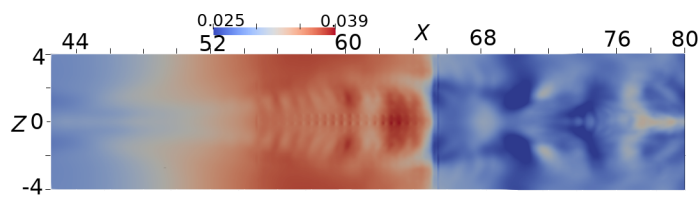

(a) pressure field

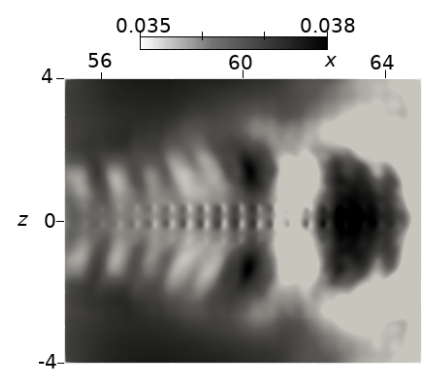

(b) Close-up on porous surface

Figure 13: Pressure field (a) and close-up on the porous surface with grey-scale (b). Top view

Figure 14 shows the near-wall streamwise-velocity streaky structures formed 470 in the downstream transition region, whereas figure 15 shows patterns of the transition process at the height $y=1$ within the boundary layer, for streamwise velocity, temperature and Schlieren image. A violent and rapid transition process is observed, which occurs through nonlinear breakdown of the side streaks first (downstream of $x=70$ ), followed by breakdown and final fragmentation of the main mushroom-structure along the midspan axis, downstream of $x=100$.

Figure 16 shows a Schlieren 3D view, on different cross-sections along the streamwise direction. The streamwise evolution of the early vortical structures and the main mushroom structure can be observed, along with their downstream fragmentation and randomisation leading to a turbulent state.

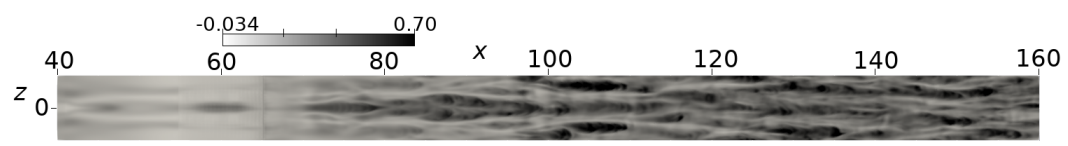

Figure 14: Near-wall streamwise velocity. Top view 


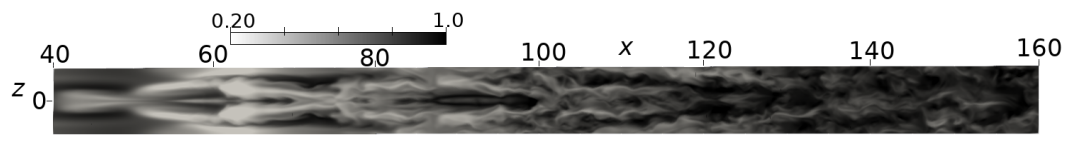

(a) $u$

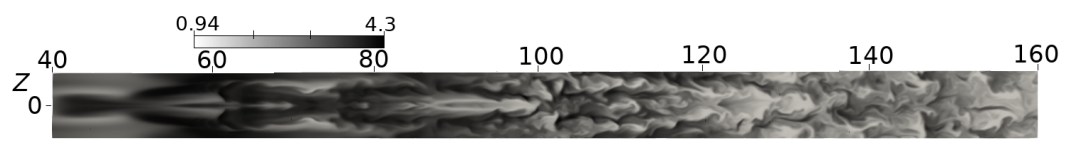

(b) $T$

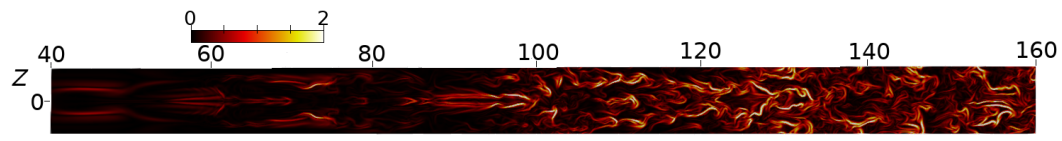

(c) Schlieren

Figure 15: Streamwise velocity (a), temperature (b), and Schlieren at $y=1$. Top view

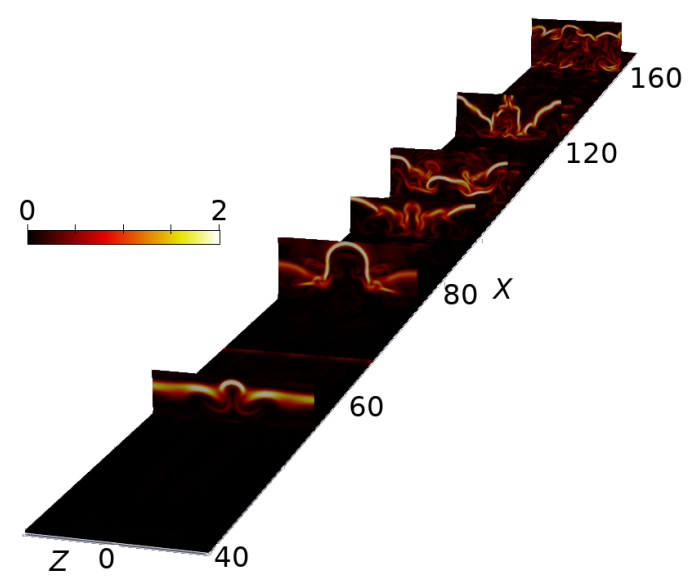

Figure 16: 3D view of Schlieren

\subsection{Effect on cooling performance and comparison with slot injection}

We analyse the cooling performance in terms of the coolant distribution within the boundary layer, the near-wall temperature and the cooling effectiveness on the wall. Figure 17 shows the coolant concentration on the $x y$-plane at $z=0$, i.e. at the midspan location. As can be seen, the coolant concen- 


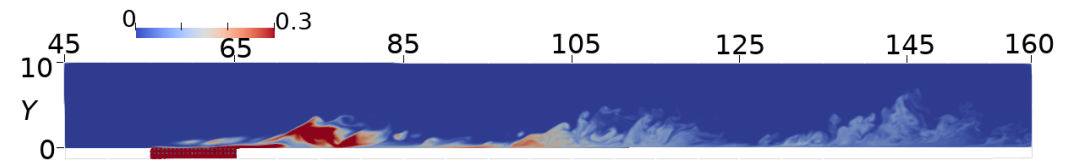

Figure 17: Coolant concentration in the $x y$-plane at $z=0$

Figure 18 shows a comparison between the porous injection and the slot injection case shown in 37, corresponding to the lowest experimental slot blowing ratio $\left(F_{\text {slots }}=0.045\right)$ considered in [36], relative to the coolant concentration at the height $y=1$ within the boundary layer.

In 37] numerical results for the wall heat flux were compared to the corresponding experimental data at a specific sensor location for different blowing ratios. The comparison showed a good agreement between numerical and experimental results for all the blowing ratios when disturbances of moderate amplitude (order of $10^{-3}$ ) are introduced within the boundary layer, corresponding to a perturbed state (not yet transitional) of the boundary layer. In the present work, in contrast, we are considering higher amplitude disturbances, which induce early transition to turbulence, in order to predict the effect of porous injection on the transition process and the resulting cooling performance.

The simulated slot injection and porous injection cases have a different blow505 ing ratio condition, but are, in contrast, comparable with respect to the total mass discharge rate over the entire injecting area $\left(d=\rho U A_{i n j}\right)$. The latter is obtained from the simulations as the product between the average blowing ratio over the blowing surface and the effective injecting area. Similar values of the mass flow discharge, namely $d_{\text {slots }}=0.173$ and $d_{\text {porous }}=0.144$, have been 
obtained for the slot and porous injection cases respectively.

The same disturbance field is imposed for the porous and the slot injection cases. As mentioned in section 5.1, the porous injection, even if at a low blowing ratio, provides a more rapid transition mechanism, which can be observed from the higher degree of fragmentation and randomisation shown by the patterns of the coolant concentration for the porous injection. In addition, as a result of an enhanced turbulent mixing, a higher concentration of coolant is observed for the porous injection case along the whole domain (figure 18, which shows the imprint of the flow structures generated downstream of the injection location.

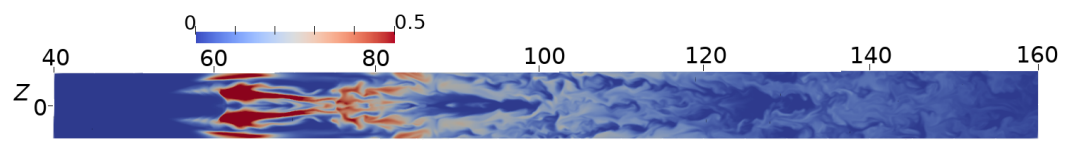

(a) porous injection

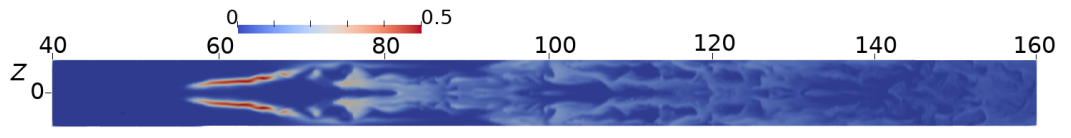

(b) slot injection

Figure 18: Coolant concentration at the height $y=1$ for porous (a) and slot (b) injection. Top view

The significant effect of porous injection on the transition mechanism is represented in figure 19, in which the Stanton number profiles, averaged in both the spanwise direction and time, are shown for the different cases of no injection, slot injection and porous injection. The Stanton number, i.e. the nondimensional surface heat flux, is defined as

$$
S t=\frac{q_{w}^{*}}{\rho_{\infty}^{*} U_{\infty}^{*} c_{p}^{*}\left(T_{a w}^{*}-T_{w}^{*}\right)},
$$

in which $q_{w}^{*}$ is the dimensional wall heat flux and $T_{a w}^{*}$ is the adiabatic wall temperature. The Stanton number profiles in figure 19 have been plotted together with the reference model profiles for a laminar and a turbulent boundary layer (represented by the blue and red dashed lines, respectively), which have been 
computed using Van Driest's correlations [27, 38].

We can observe different interesting features in three separate regions, namely an early region just downstream of the injection location, $x>65$, an intermediate region between $x=80$ and $x=110$, and a downstream region for $x>110$. In the early region, the trends for the injection cases show a lower heat flux with a much sharper rate of increase, compared to the case without injection. This is consistent with the fact that this is the immediate region downstream of the cooling injection, hence the cooling effect is a maximum, resulting in a suppression of the heat transfer rate. The sharper increase of the wall heat flux is representative of a faster early transition process associated with boundary-layer injection. In particular, the porous injection case shows the highest gradient of the Stanton number, which crosses the laminar model at approximately $x=70$ and reaches a peak higher than the other cases at $x=80$, hence demonstrating that porous injection enhances the transition process.

In the intermediate region, we can observe that all the curves collapse approximately to the same value, and increase with a similar rate, meaning that transition is proceeding at about the same rate for all the cases. This, in turn, suggests that the transition process determines the wall heat transfer rate in this region, rather than the presence of coolant within the boundary layer.

Finally, in the downstream region, we observe that the curve for the porous injection case separates from the other cases, and shows a much sharper decrease rate until it approaches the laminar model just downstream of $x=150$. This is representative of the fact that turbulent mixing dominates in this region, and is enhanced for the porous injection case, thus resulting in a significant increase of the wall cooling performance, compared to the other cases. Hence, these results demonstrate that an enhanced turbulent mixing, in the boundary layer with porous injection, provides a suppression of the wall heat flux following transition. 


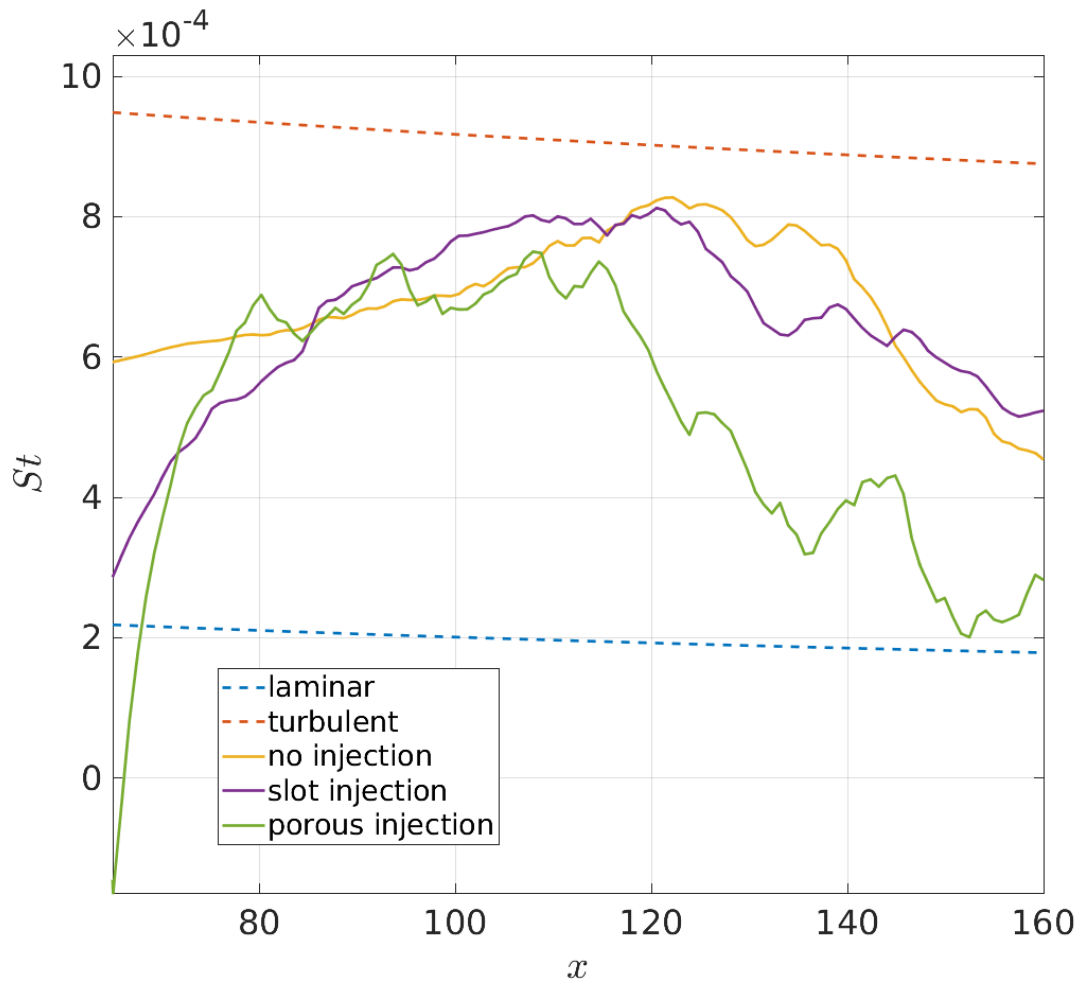

Figure 19: Stanton number along the streamwise direction, averaged both in time and in the spanwise direction

Finally, to quantify the beneficial effects of porous injection with respect to slot injection on the cooling performance, the curves of the spanwise-averaged and time-averaged cooling effectiveness along the midspan axis are shown in figure 20 for the above mentioned cases. The cooling effectiveness is computed through the formula relative to the case of an isothermal wall,

$$
\eta=1-\frac{q_{w, c}}{q_{w, n c}}
$$

where $q_{w, c}$ and $q_{w, n c}$ represent the wall heat flux with and without cooling respectively.

As can be seen from figure 20 the porous injection case provide values of the cooling effectiveness significantly higher than the slot injection case in the 
turbulent mixing region, consistent with the results for the Stanton number.

In particular, it can be seen that the overall trend of the cooling effectiveness, for both cases, is first decreasing, starting from the inital values of 0.3 and 0.4 (for porous and slot injection, respectively) reached just downstream of the injection location, and then increasing further downstream. This is due to the transition process, which is nullifying the cooling effects in the early region, consistent with the increase of the Stanton number trends shown in figure 19. The cooling effectiveness, for both cases, crosses the zero value and assumes negative values in the above-mentioned intermediate region (with a minimum of approximately -0.1), where the transition process determines the wall heat transfer rate, producing the aeroheating effect (hence negative cooling effectiveness). Then, in the downstream region, where the turbulent mixing effect dominates, the cooling effectiveness shows a sharp increase for both cases, with the highest rate and maximum value $(\eta \approx 0.6)$ observed for the porous injection case. 


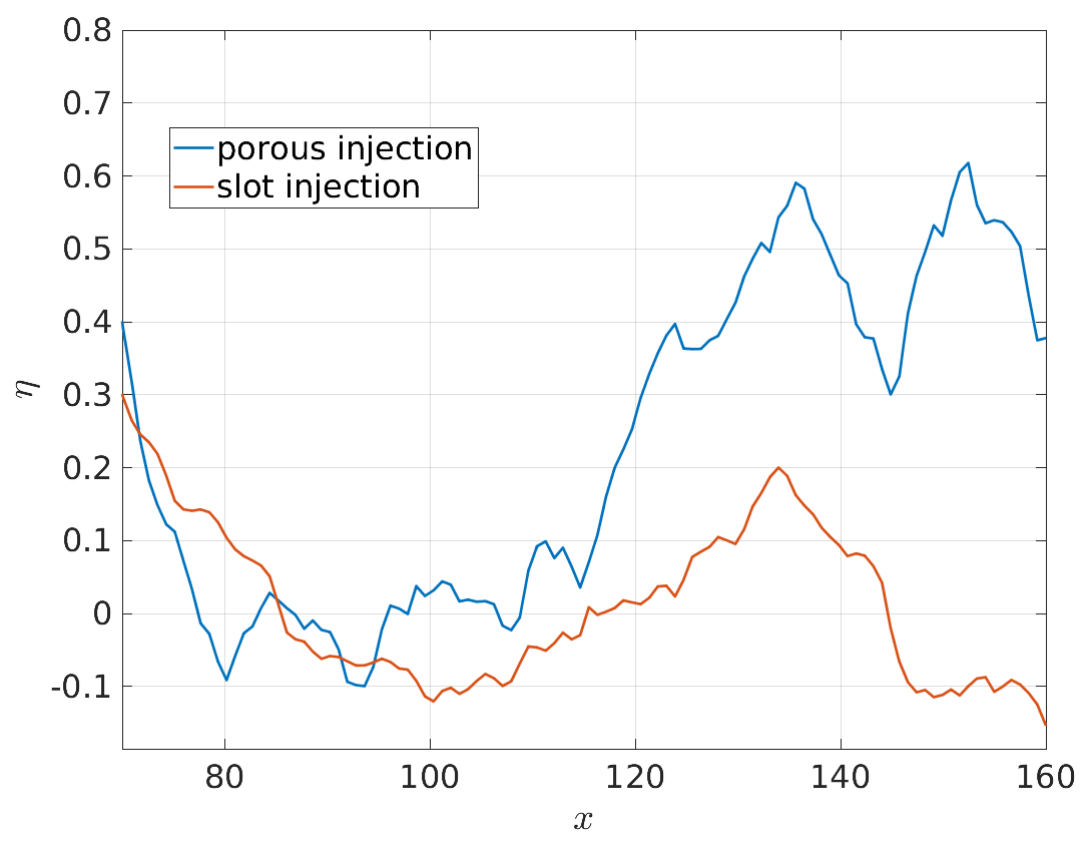

Figure 20: Cooling effectiveness along the streamwise direction, averaged both in time and in the spanwise direction

In conclusion, we have seen that inserting high-amplitude disturbances associated with the most unstable 3D first mode waves into the boundary layer with porous injection has a strong effect on both the transition process and the wall cooling performance. A rapid transition process of the first mode, with evidence of nonlinear secondary instabilities and streak breakdown, has been observed, which results in a higher cooling effectiveness, associated with a more efficient turbulent mixing in the downstream region. However, it should be mentioned that these results are associated with a high amplitude (order of $10^{-2}$ ) of the imposed boundary-layer disturbances, hence to the presence of nonlinear effects. In order to assess the effect of porous injection on the growth of boundary-layer instabilities and the resulting cooling performance on the plate surface in a linear regime, it would be necessary to simulate disturbance amplitudes of lower order of magnitude. Future studies based on different amplitudes of the forcing disturbances, carried out over a broad range of blowing ratios, would provide a 
full spectrum of the effect of porous injection on boundary-layer stability and transition, as well as on the associated cooling performance, which is very important for the assessment of transpiration cooling systems in hypersonic flows.

\section{Conclusion}

The present work has presented and assessed a mesoscopic modelling approach designed for the prediction of an equivalent Darcy-Forchheimer behaviour, in the context of the flow through a porous medium, that mimics a real porous material sample through a porous layer with higher inner pore length scales. The aim of this methodology is to enable cost-efficient direct numerical simulations of injection through a porous structure in a hypersonic flow for transpiration cooling applications. The simulations have been based on experiments carried out in a hypersonic wind tunnel at Mach 5, with air injection through a sample of porous UHTC material, with a porosity of $42 \%$, and an inner pore length scale of the order of 1 to $4 \mu \mathrm{m}$.

The methodology, based on former computational studies, has been extended to incorporate a rescaling of the pore size from the realistic micrometer length scales to higher and more affordable length scales, imposing the aerodynamic similarity between different cases with the same porosity and the same characteristic Reynolds number $R e_{c}$. Results of simulations carried out for higher cylinder diameters, namely $24 \mu \mathrm{m}, 48 \mu \mathrm{m}$ and $96 \mu \mathrm{m}$, have shown that, by keeping constant the characteristic Reynolds number $R e_{c}$, and by opportunely rescaling the pressure drop with the imposed pore size $L$ through a derived formula, an equivalent Darcy-Forchheimer behaviour to the reference experimental microstructure can be obtained.

The porous layer approach with staggered spheres has been applied to a 3D large domain case of porous injection over a flat plate, to study the transition mechanism and the cooling performance in comparison with a reference case of slot injection. Results of the direct numerical simulations have shown that porous injection leads to a more rapid transition to turbulence, compared to 
slot injection. These pressure waves, which are of larger scale than the spheres representing the porous surface, promote nonlinear breakdown of the streaks via generation of secondary instabilities. The more rapid transition process observed for the porous injection case, in turn, enhances the turbulent mixing and a higher distribution of the coolant within the boundary layer. This has the beneficial effect of increasing the cooling performance by reducing the temperature near the wall, which provides a higher cooling effectiveness, compared to the slot injection case.

\section{Acknowledgements}

The authors would like to acknowledge support from EPSRC (Engineering and Physical Sciences Research Council) under the Grant No. EP/P000878/1.

[1] A. Fitt, J. Ockendon, T. Jones, Aerodynamics of slot-film cooling: theory and experiment, Journal of fluid mechanics 160 (1985) 15-27.

[2] A. Fitt, P. Wilmott, Slot film coolingthe effect of separation angle, Acta mechanica 103 (1-4) (1994) 79-88.

[3] S. Wittig, A. Schulz, M. Gritsch, K. A. Thole, Transonic film-cooling investigations: effects of hole shapes and orientations, in: ASME 1996 International Gas Turbine and Aeroengine Congress and Exhibition, American Society of Mechanical Engineers, 1996, pp. V004T09A026-V004T09A026.

[4] S. Baldauf, A. Schulz, S. Wittig, High-resolution measurements of local heat transfer coefficients from discrete hole film cooling, Transactions of the ASME-T-Journal of Turbomachinery 123 (4) (2001) 749-757.

[5] J. Meinert, J-ograve, r. Huhn, E. Serbest, O. J. Haidn, Turbulent boundary layers with foreign gas transpiration, Journal of Spacecraft and Rockets 38 (2) (2001) 191-198. 
[6] T. Langener, J. V. Wolfersdorf, J. Steelant, Experimental investigations on transpiration cooling for scramjet applications using different coolants, AIAA journal 49 (7) (2011) 1409-1419.

[7] K. Heufer, H. Olivier, Experimental and numerical study of cooling gas injection in laminar supersonic flow, AIAA journal 46 (11) (2008) 27412751.

[8] M. A. Keller, M. J. Kloker, H. Olivier, Influence of cooling-gas properties on film-cooling effectiveness in supersonic flow, Journal of Spacecraft and Rockets 52 (5) (2015) 1443-1455.

[9] M. A. Keller, M. J. Kloker, Direct numerical simulation of foreign-gas film cooling in supersonic boundary-layer flow, Aiaa Journal 55 (1) (2016) 99111.

655 [10] S. Ergun, A. A. Orning, Fluid flow through randomly packed columns and fluidized beds, Industrial \& Engineering Chemistry 41 (6) (1949) 11791184 .

[11] I. Macdonald, M. El-Sayed, K. Mow, F. Dullien, Flow through porous media-the ergun equation revisited, Industrial \& Engineering Chemistry Fundamentals 18 (3) (1979) 199-208.

[12] E. Erdim, Ö. Akgiray, İ. Demir, A revisit of pressure drop-flow rate correlations for packed beds of spheres, Powder technology 283 (2015) 488-504.

[13] N. R. Wilson, R. H. Shaw, A higher order closure model for canopy flow, Journal of Applied Meteorology 16 (11) (1977) 1197-1205.

[14] M. R. Raupach, R. Shaw, Averaging procedures for flow within vegetation canopies, Boundary-Layer Meteorology 22 (1) (1982) 79-90.

[15] J. Wilson, A second-order closure model for flow through vegetation, Boundary-Layer Meteorology 42 (4) (1988) 371-392. 
[16] Z. Li, J. Lin, D. Miller, Air flow over and through a forest edge: a steadystate numerical simulation, Boundary-Layer Meteorology 51 (1-2) (1990) 179-197.

[17] H. Wang, E. S. Takle, Boundary-layer flow and turbulence near porous obstacles, Boundary-Layer Meteorology 74 (1-2) (1995) 73-88.

[18] T. Masuoka, Y. Takatsu, Turbulence model for flow through porous media, International Journal of Heat and Mass Transfer 39 (13) (1996) 2803-2809.

[19] M. J. de Lemos, M. H. Pedras, Recent mathematical models for turbulent flow in saturated rigid porous media, J. Fluids Eng. 123 (4) (2001) 935-940.

[20] J. Kozeny, Uber kapillare leitung der wasser in boden, Royal Academy of Science, Vienna, Proc. Class I 136 (1927) 271-306.

[21] A. W. Heijs, C. P. Lowe, Numerical evaluation of the permeability and the kozeny constant for two types of porous media, Physical Review E 51 (5) (1995) 4346.

[22] P. C. Carman, Fluid flow through granular beds, Chemical Engineering Research and Design 75 (1997) S32-S48.

[23] M. Matyka, A. Khalili, Z. Koza, Tortuosity-porosity relation in porous media flow, Physical Review E 78 (2) (2008) 026306.

[24] O. Coulaud, P. Morel, J. Caltagirone, Numerical modelling of nonlinear effects in laminar flow through a porous medium, Journal of Fluid Mechanics 190 (1988) 393-407.

[25] S. Lee, J. Yang, Modeling of darcy-forchheimer drag for fluid flow across a bank of circular cylinders, International journal of heat and mass transfer 40 (13) (1997) 3149-3155.

[26] N. Jeong, D. H. Choi, C.-L. Lin, Prediction of darcy-forchheimer drag for micro-porous structures of complex geometry using the lattice boltzmann 
method, Journal of Micromechanics and Microengineering 16 (10) (2006) 2240.

[27] J. D. Anderson, Hypersonic and High-Temperature Gas Dynamics, Second Edition, AIAA Education Series, 2006.

[28] P. D. Neufeld, A. Janzen, R. Aziz, Empirical equations to calculate 16 of the transport collision integrals $\omega(1, \mathrm{~s})^{*}$ for the lennard-jones $(12-6)$ potential, The Journal of Chemical Physics 57 (3) (1972) 1100-1102.

[29] D. J. Hill, D. I. Pullin, Hybrid tuned center-difference-weno method for large eddy simulations in the presence of strong shocks, Journal of Computational Physics 194 (2) (2004) 435-450.

[30] C. Pantano, R. Deiterding, D. J. Hill, D. I. Pullin, A low numerical dissipation patch-based adaptive mesh refinement method for large-eddy simulation of compressible flows, Journal of Computational Physics 221 (1) (2007) 63-87.

[31] J. L. Ziegler, R. Deiterding, J. E. Shepherd, D. I. Pullin, An adaptive highorder hybrid scheme for compressive, viscous flows with detailed chemistry, Journal of Computational Physics 230 (20) (2011) 7598-7630.

[32] M. Ihme, Y. Sun, R. Deiterding, Detailed simulations of weak-to-strong ignition of a h 2/o 2/ar mixture in shock-tubes, in: International Symposium on Shock Waves, Springer, 2013, pp. 209-214.

[33] A. Cerminara, R. Deiterding, N. Sandham, Dns of hypersonic flow over porous surfaces with a hybrid method, in: 2018 AIAA Aerospace Sciences Meeting, 2018, p. 0600.

[34] A. Cerminara, R. Deiterding, N. Sandham, Dns of hypersonic flow over porous surfaces with a hybrid method, in: 2018 AIAA Aerospace Sciences Meeting, 2018, p. 0600. 
[35] R. Deiterding, Construction and application of an amr algorithm for distributed memory computers, in: Adaptive Mesh Refinement-Theory and Applications, Springer, 2005, pp. 361-372.

[36] T. Hermann, H. S. Ifti, M. McGilvray, L. Doherty, R. P. Geraets, Mixing characteristics in a hypersonic flow around a transpiration cooled flat plate model, in: HiSST: International Conference on High-Speed Vehicle Science Technology, Moscow, Russia, 2018.

[37] A. Cerminara, R. Deiterding, N. Sandham, Dns of injection-induced transition in hypersonic flow over porous surfaces for transpiration cooling applications, in: 11th International Symposium on Turbulence and Shear Flow Phenomena (TSFP11), 2019.

[38] E. R. Van Driest, The problem of aerodynamic heating, Institute of the Aeronautical Sciences, 1956. 